\title{
BMJ Open Mindfulness-based stress reduction (MBSR) as sole intervention for non- somatisation chronic non-cancer pain (CNCP): protocol for a systematic review and meta-analysis of randomised controlled trials
}

\author{
Lawrence Leung, ${ }^{1,2,3}$ Han Han, ${ }^{2}$ Mary Martin, ${ }^{2}$ Jyoti Kotecha ${ }^{2}$
}

To cite: Leung L, Han $\mathrm{H}$, Martin $\mathrm{M}$, et al. Mindfulnessbased stress reduction (MBSR) as sole intervention for non-somatisation chronic non-cancer pain (CNCP): protocol for a systematic review and meta-analysis of randomised controlled trials BMJ Open 2015;5:e007650. doi:10.1136/bmjopen-2015007650

- Prepublication history and additional material is available. To view please visit the journal (http://dx.doi.org/ 10.1136/bmjopen-2015007650).

Received 12 January 2015 Revised 20 April 2015 Accepted 23 April 2015

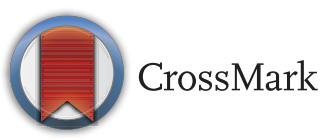

${ }^{1}$ Department of Family Medicine, Queen's University, Kingston, Ontario, Canada ${ }^{2}$ Centre of Studies in Primary Care, Queen's University, Kingston, Ontario, Canada

${ }^{3}$ School of Medicine, Queen's University, Kingston, Ontario, Canada

Correspondence to Professor Lawrence Leung; leungl@queensu.ca

\section{ABSTRACT}

Introduction: Chronic non-cancer pain (CNCP) affects up to $50 \%$ of the world's population. It impacts negatively on quality of life; entailing high costs on our medical systems, and translates to economic burden due to work loss. Aetiology of CNCP is complex and multifactorial, embracing the somatosensory, cognitive and affective domains. Opioid analgesia and other invasive interventions are often inadequate for clinical management of CNCP. Recently, mindfulness-based stress reduction (MBSR) has become a popular therapy for various medical conditions, including CNCP. However, studies reported varying efficacies, and relevant systematic reviews have included clinical trials with inherent heterogeneity either in study conditions or types of interventions used. Our study aims to provide an updated and more critical evaluation of the efficacy of MBSR as the intervention for non-somatisation CNCP.

Methods and analysis: A systematic review with meta-analysis of randomised controlled trials published in English will be performed in accordance with the Preferred Reporting Items for Systematic reviews and Meta-analyses (PRISMA) guidelines and the Cochrane Collaboration format. MEDLINE, EMBASE, PsychINFO, and the Cochrane Central Register of Controlled Trials Intervention, will be searched independently by reviewers using defined MeSH terms. Studies with full texts using MBSR as the main intervention on patients with non-somatising CNCP will be included. Outcome measures include pain scores and disability assessment scales. Continuous data will be meta-analysed using the RevMan 5 Review Manager programme. Primary analysis will adopt the random effects model in view of heterogeneity between trials. The standardised mean difference will be expressed as the effect size with $95 \%$ Cls. Forest plots, funnel plots, the $\mathrm{I}^{2}$ statistic and the Cochrane Risks of Bias Assessment table will be included.

Ethics and dissemination: No ethics approval is deemed necessary. Results of this study will be disseminated via peer-reviewed publications and scientific meetings.

\section{Strengths and limitations of this study}

- Compared with similar publications, our study aims to provide an update and more critical evaluation on the critical efficacy of mindfulnessbased stress reduction (MBSR) as the main intervention for non-somatising chronic noncancer pain

- The study will provide useful evidence-based guidance for healthcare providers and policy stakeholder to facilitate the option of MBSR for appropriate patients with chronic pain.

- Our results may be limited by heterogeneity from smaller trials.

Trials registration number: PROSPERO CRD42014015568.

\section{INTRODUCTION}

Chronic non-cancer pain and its treatment Chronic non-cancer pain (CNCP) refers to pain of non-malignant aetiology that lasts for more than 3 months. It is a condition commonly seen in any population which is often clinically challenging to manage. Worldwide prevalence of CNCP ranges from $10.1 \%$ to $55.2 \%,{ }^{1}$ and in the USA, CNCP affects more than 100 million people, entailing combined direct and indirect costs of US $\$ 635$ billion annually. ${ }^{2}$ In Canada, it has been estimated that one in five Canadians are afflicted with CNCP. ${ }^{3} 4$ A questionnaire study reported CNCP prevalence of $55-72 \%$ among the East London dwellers. ${ }^{5}$ Using a Human Development Index of 0.9 as the watershed between developed $(\geq 0.9)$ and developing countries $(\leq 0.9)$, a 
recent systematic review of studies showed that CNCP is more prevalent in developing countries. ${ }^{6}$ The whole list of CNCP diagnoses runs long, with the leading four being osteoarthritis, low back pain, headaches and neuropathic pain. ${ }^{2}$ The latest consensus describes CNCP as a neuro-signature which is automatically generated within the central nervous system, at an intensity depending on net interactions between the somatosensory, cognitive and affective domains. ${ }^{78}$ Opioids have widely been used by clinicians to treat CNCP, despite divergent opinions and cautionary notes on their efficacies and indications. ${ }^{2}$ An initial Cochrane review in 2007 did not support evidence of using opioids for chronic low back pain. ${ }^{9}$ However, such opinion was reversed by Cochrane in 2013, showing short-term efficacy of opioids for chronic low back pain. ${ }^{10} 11$ Thus said, patients with CNCP are often not effectively treated, with up to $40 \%$ having uncontrolled pain. ${ }^{4}$ Effective management for CNCP should extend the classic thinking of the analgesic ladder described by $\mathrm{WHO}^{12}$ to a broader analgesic platform, ${ }^{8}$ incorporating non-pharmacological treatment options, such as physiotherapy, acupuncture, chiropractic, mindfulness-based therapy, cognitive behaviour therapy, relaxation, yoga and other mind-body therapies. Recent systematic reviews found favourable evidence of nonpharmacological modalities for CNCP in both general ${ }^{13}$ and elder populations. ${ }^{14}$ Among these options, mindfulness-based stress reduction (MBSR) therapy, a form of mind-body therapy, has been widely advocated for patients with CNCP.

\section{Description of the intervention}

First described by Kabat-Zinn ${ }^{15}$ as an out-patient programme for patients with various conditions of chronic pain that could not be treated effectively within hospital, MBSR therapy combines meditation, body-awareness and yoga to enhance the individual's ability to selfregulate and hence cope with the pain experience. The two kernels of MBSR are mindfulness and meditation. Kabat-Zinn defined mindfulness as intentional, nonjudgmental and accepting awareness and focus of oneself', and meditation as 'disciplinary self-regulation of attention from moment to moment'. ${ }^{15}$ Combined, mindfulness meditation is a practice with ancient roots in Theravada Buddhism (known as sattipatana vipassana) and Mahayana Buddhism and yoga. ${ }^{15}$ The original programme described by Kabat-Zinn consists of 10 weekly lessons each lasting $2 \mathrm{~h}$, where three practices will be taught: (1) total body scan (sweeping) from head to toes, where the individuals lie supine and regulate their attention to body sensation and relaxed breathing; (2) mindfulness of sensation and breathing where the individuals sit in a chair and focus intentionally and nonjudgmentally on themselves and (3) Hatha yoga postures where the individuals meditate to be detached and observe their inner emotions and thinking processes such that if they drift, the individuals will direct attention to themselves from moment to moment. In the first
4 weeks, sweeping and mindfulness will be taught with supplementary audio-cassette tape instructions for daily practices. In the next 4 weeks, Hatha yoga will be added to intercalate with sweeping, to be practised daily aided by audio-cassette tape instructions. From weeks 9-10, individuals will be allowed to practise any routines previously taught for 30-45 min a day. Compared with controls, Kabat-Zinn found that individuals who went through the MBSR programme had significant reduction in pain, negative body image, mood disturbance, analgesics requirement with an increase in self-esteem and physical activity levels. Such improvements were sustained through 15 months of post-treatment. ${ }^{16}$ Soon after, MBSR evolved to be a main-stream patient-centred curriculum hosted by the University of Massachusetts, consisting of an 8 -week programme with a weekly $2 \frac{1 / 2}{2} \mathrm{~h}$ class and one all-day class, totally $31 \mathrm{~h}$ of instruction. Similar to the original programme that Kabat-Zinn used in 1982, the content comprises instructions on mindfulness, meditation and yoga techniques supplemented by audio-visual guidance and home practice. ${ }^{17}$ This format of MBSR is often adopted by clinical studies and trials as the standard regime, referred as the Kabat-Zinn protocol. It also becomes popular, globally, that interested people can be trained to become certified MBSR instructors in over 30 countries. ${ }^{18}$ Not surprisingly, the upsurge of MBSR practice aroused intense research interests and led to a 10-fold jump in annual publications in this topic from year 2003 to 2012. ${ }^{18}$

\section{How the intervention might work}

\section{Psychological theory}

The current belief states that MBSR offers benefits by regulating attention to present moment awareness of the body and emotions, acknowledging their changes and shifts in an observant and non-judgemental manner. This results in a state of equanimity in the body and mind which will alleviate and even remove all pain and physical sufferings in the mind-body continuum. ${ }^{15} 1619$ The net outcomes include direct reduction of stress and improvement of mood, hence translating to better tolerance of pain and increased exercise tolerance, hence enhanced quality of life. ${ }^{16}$

\section{Biological markers}

Scientists have reported biological markers as surrogate measures for the therapeutic effects of MBSR. Physiologically, serum cortisol level is a quantifiable measure of stress. In two separate studies, Carlson $e t a l^{20}{ }^{21}$ demonstrated significant reduction of salivary cortisol concentration after participation in the MBSR programme with matching improvement in symptoms of stress and sleep patterns. Another hypothesis states that MBSR exerts its positive effects via modulation of the immune system. However, results from studies have not been conclusive. ${ }^{22}{ }^{23}$ Other immune and proinflammatory markers being implicated for the therapeutic mechanisms of MBSR-included serum natural killer 
cells, ${ }^{24} \mathrm{C}$ reactive protein ${ }^{25}$ and gene expression of the $\mathrm{NF}-\kappa \mathrm{B}$ (nuclear factor $\kappa$-light-chain-enhancer of activated $B$ cells) ${ }^{25}$

\section{fMRI neuroimaging}

Functional MRI (fMRI) is a real-time neuroimaging technique which detects changes of oxygenation within neuronal tissues as a result of neural activities, hence identifying neuroanatomical substrates that were active within the defined time. Using fMRI, MBSR has been shown to alter the functional connectivity between areas of the brain that are known to control attentional focus and sensory processing. ${ }^{26}$ MBSR can also alleviate negative emotions aroused by unpleasant stimuli, ${ }^{27}$ or, directly inhibit the brain areas that respond to aversive stimuli. $^{28}$ One study even showed that a standard 8 weeks MBSR therapy improved mild cognitive impairment in patients with Alzheimer's disease. ${ }^{29}$

\section{EEG and magnetoencephalography}

In parallel with fMRI findings, studies have shown MBSR leads to recongised patterns of change in EEG activities. Again, there is lack of consensus. ${ }^{30-32}$ Using more advanced technology of magnetoencephalography (MEG), similar patterns of signal enhancement have been found after MBSR practice, in particular areas of the brain that modulate attention and somatosensory reception of the body. ${ }^{33}$ However, MEG recordings are always challenged by issues of sensitivities and stray signals, and positive changes in patterns may not be localising enough for meaningful neuro-anatomical correlation.

\section{Why is it important to do this review?}

Two systematic reviews had been published which looked at use of MBSR for CNCP. ${ }^{35}{ }^{36}$ Analysis of the clinical trials that were included showed a certain level of heterogeneity in terms of the actual intervention used, the conditions of chronic pain, and the outcome measures. In particular, the inclusion of fibromyalgia and chronic fatigue syndrome under the category of chronic pain will inevitably confound the results due to possible somatisation variables. Also, a number of relevant controlled clinical trials have been published since 2011 which were not included in the reviews by Teixeira ${ }^{35}$ and Veehof et al..$^{36}$ This forms the basis of this proposed systematic review and meta-analysis (table 1 ).

\section{OBJECTIVES}

The primary objective of this systematic review is to provide an updated evaluation of the efficacy of MBSR as the sole intervention for non-somatisation types of CNCP.

\section{METHODS AND ANALYSES}

A systematic review and meta-analysis will be performed according to the format specified in the Cochrane
Handbook for Systematic Reviews of Intervention. ${ }^{37}$ The logistics and reporting will conform to the Preferred Reporting Items for Systematic Reviews and Meta-analyses (PRISMA) statements. ${ }^{38}$ Quantitative data derived from the randomised trials as published in the included studies will be systematically reviewed and meta-analysed.

\section{Criteria for considering studies for review Types of studies}

This review will only include fully published randomised controlled trials (RCTs) (either in full scale or as pilot, irrespective of blinding status) that look at the effects of MBSR in the treatment of CNCP. MBSR must be given as the sole intervention, lasting at least six sessions, delivered either face-to-face or by online mode. The control group can be either passive (ie, watchful waitlist) or active (receiving other standard care or pain management). Quasi-RCTs will not be included.

\section{Types of participants}

Participants of either gender, aged 16years or above, having a form of chronic pain (specified or not) of more than 3 months' duration, which is not related to a cancer or somatisation diagnosis will be eligible. Patients with known psychiatric disorders, taking medications or undergoing active interventions for chronic pain will be excluded.

\section{Types of interventions}

MBSR must be given as the sole intervention lasting at least six sessions, delivered either face-to-face or by online mode. The control group can be either passive (ie, watchful waitlist) or active (receiving other standard care or pain management).

\section{Types of outcome measures}

The following outcome measures will be evaluated basing them on reported data analysis accrued from individuals in each included trial:

- Primary outcome

Pain scores either as visual analogue scale or numeric pain score

\section{- Secondary outcome}

1. General well-being and quality of life-SF-36, SF-12;

2. Disability scores -Pain Catastrophizing Scale, Roland Morris Disability Questionnaire.

\section{Search methods and strategies \\ Electronic searches}

Two reviewers (LL and $\mathrm{HH}$ ) will perform an electronic search using the following databases from the OvidSP portal of the Queen's University:

- MEDLINE (1946 to 9 November 2104);

- EMBASE Classic+EMBASE (1947 to 9 November 2014);

- PsychINFO (1967 to 9 November 2014); 


\begin{tabular}{|c|c|c|c|c|}
\hline Review & $\begin{array}{l}\text { Number of studies } \\
\text { included }\end{array}$ & $\begin{array}{l}\text { Pain conditions } \\
\text { (number of studies) }\end{array}$ & $\begin{array}{l}\text { Interventions used } \\
\text { (number of studies) }\end{array}$ & $\begin{array}{l}\text { Outcome measures } \\
\text { (number of studies) }\end{array}$ \\
\hline $\begin{array}{l}\text { Veehof } \\
\text { et } a l^{\beta 6}\end{array}$ & 22: $9 \mathrm{RCT}+13 \mathrm{CT}$ & $\begin{array}{l}10 \text { Chronic pain } \\
4 \text { Fibromyalgia } \\
4 \text { Chronic fatigue } \\
\text { Syndrome } \\
2 \text { Rheumatoid arthritis } \\
1 \text { Chronic headache } \\
1 \text { Whiplash injury }\end{array}$ & $\begin{array}{l}14 \text { MBSR } \\
7 \text { ACT } \\
1 \text { MBSR+Qigong }\end{array}$ & $\begin{array}{l}15 \text { use a pain score with } \\
\text { depression/QoL scores } \\
7 \text { use depression/QoL scores } \\
\text { only }\end{array}$ \\
\hline Teixeira $^{35}$ & 10: $4 \mathrm{RCT}+6 \mathrm{CT}$ & $\begin{array}{l}7 \text { Chronic pain } \\
2 \text { Fibromyalgia } \\
1 \text { chronic headache }\end{array}$ & $\begin{array}{l}7 \text { MBSR } \\
1 \text { MBSR+aromatherapy } \\
2 \text { meditation }\end{array}$ & $\begin{array}{l}9 \text { use a pain score with } \\
\text { depression/QoL scores } \\
1 \text { use depression/QoL scores } \\
\text { only }\end{array}$ \\
\hline
\end{tabular}

ACT, acceptance and commitment therapy; CT, controlled trial; MBSR, mindfulness-based stress reduction; QoL, quality of life; RCT, randomised controlled trial.

- Cochrane Central Register of Controlled Trials (up to and including October 2014).

The following MeSH terms will be used: 'randomised', 'randomized', 'controlled trials', 'chronic pain', 'mindfulness' and 'mindfulness-based stress reduction'. After selecting the four databases, sequential searches using each MeSH term as a keyword will be made, which will then be concatenated with BOOLEAN operators as a final search string, as follows:

(((('Mindfulness-based stress reduction)' OR 'mindfulness)') $A N D$ ((randomised) OR randomized)) AND (('chronic pain)' $A N D$ 'controlled trial)'.

Search for earlier records will not be attempted, as mindfulness-based interventions did not come into existence before 1946. Owing to different workplace locations of LL and $\mathrm{HH}$, non-identical computers will be employed but identical search protocols will be used via the same gateway from the URL link on the website of Queen's Health and Life Sciences Database Services. ${ }^{39}$

\section{Other searches}

Results from this initial search will be supplemented by additional records from bibliography lists of relevant and index review papers as mentioned in the previous sections. Grey literature will also be searched via the 'GREY MATTERS' checklists from the Canadian Agency for Drugs and Technologies in Health $(\mathrm{CADTH}) .{ }^{40}$ If deemed appropriate, conference proceedings and correspondence with experts in the field will also be considered.

\section{Logistics and output}

Using the PRISMA flow diagram for reporting systematic reviews and meta-analyses (see online supplementary appendix A), ${ }^{38}$ all pooled records will be de-duplicated with additional filters added. Non-English studies and records without full texts will be excluded, so also studies that are irrelevant to the context. The two reviewers will compare their search results, and if mutual concordance is within $95 \%$, the records will be accepted with the contested records included.

\section{Data collection and analysis}

\section{Selection of studies}

Basing on the retrieved titles and abstracts, three reviewers (LL, HH and MM) will screen the studies according to the inclusion and exclusion criteria as listed above. If in doubt, the full text of the study will be retrieved either via online or through the help of Queen's University Librarian at the Bracken Library. All reviewers will meet at least twice to discuss and compare their verdicts. If in doubt, full texts of the articles will be retrieved for more detailed examination. Discrepancies and inconsistencies will be discussed and reconciled. An inter-rater concordance of $95 \%$ will be targeted before moving onto the next stage. Any contested record(s) will not be included for data extraction.

\section{Data extraction and assessment for relevance}

After finalising the studies for meta-analyses, two reviewers (LL and $\mathrm{HH}$ ) will perform data extraction for each study using two tools: (1) the Cochrane Data Collection Form (RCTs only) ${ }^{41}$ and (2) the Review Manager (RevMan) software V.5.3.5. ${ }^{42}$ Data will be entered as per the following domains:

- For methods, the design of study (randomised or non-randomised, pilot or full);

- For participants, the total number with basic characteristics, pain conditions, their allocation groups and sizes with randomisation protocol (if appropriate);

- For interventions, details of intervention in nature and duration versus control;

- For outcomes, the drop-out versus completion rate, time point(s) of measurement, the tool(s) used and modality of final analysis (per-protocol vs intention-to-treat). 
Where there is more than one measure outcome for each study, data will be entered separately for each outcome to enable individual analysis. In this meta-analysis, the default data type will be continuous data expressed in means and SD for participants in the intervention versus control group. Data in other forms will be converted accordingly to enable pooled analysis. Two other reviewers (JK and MM) will revise the accuracy of the extracted data, and any disagreement and discrepancies will again be resolved by consensus meetings and additional consultation with our in-house statistician.

\section{Risks of bias assessment}

The methodological vigour and quality of each study will be evaluated with the Cochrane Risk of Bias Assessment tool $^{37} 43$ which is an integral part of the Cochrane Data Extraction Form and the RevMan programme. Here six domains of bias (selection, performance, detection, attrition, reporting and other) will be assessed, each to be graded as either 'low-risk', 'unclear-risk' or 'high-risk' with juxtaposed commentary space to support such rating. ${ }^{43}$

\section{Data analysis and synthesis}

Unit of analysis

Each individual in every included trial as randomised to any arm will be counted only once as a single unit. Where possible, the same measurement time point will be used across different studies. If not possible, the nearest time point data will be adopted.

\section{Measurement of treatment effect}

The RevMan 5.3.5 programme will be used as the principal tool for meta-analysis. The random effects model will be chosen instead of the fixed effects model, in view of the potential yet uncontrollable heterogeneity of diagnoses that were categorised as 'chronic pain' in the included studies. For each measure of outcome, continuous data as expressed in means from included studies will be extracted and analysed, generating a standardised mean difference (SMD) as the effect size with 95\% CIs. The Hedges $\mathrm{g}$ statistic, as described by Hedges and Olkins, ${ }^{44}$ is the default formulation adopted by the RevMan programme to derive the SMD ${ }^{45}$ Where appropriate, the weighted mean difference will also be quoted for analysis.

\section{Data integrity and dealing with missing data}

Two reviewers (MM and JK) will be responsible for error checking of all text and quantitative data as entered by the other two reviewers (LL and $\mathrm{HH}$ ). In case of missing, incomplete or equivocal data, efforts will be made to contact the authors of published trials for clarifications and advice.

\section{Assessment of heterogeneity}

To assess heterogeneity of studies, the $\tau^{2}, \chi^{2}$ and $\mathrm{I}^{2}$ statistics will be reported in parallel with the SMDs. The $\mathrm{I}^{2}$ statistic will be chosen as the reference measure for comparison. By common convention, a value of $\leq 25 \%$ indicates low heterogeneity; $\mathrm{I}^{2} \leq 50 \%$ indicates moderate heterogeneity, and $\mathrm{I}^{2} \geq 75 \%$ indicates high heterogeneity. ${ }^{46}$ Where necessary, Forest plots and heterogeneity funnel plots will be generated for visual presentation. Funnel plots are useful as a quick screen for publication biases, which are often found in smaller studies that report significant effects in their outcomes. Thus said, funnel plots, per se, are not specific for publication biases. $^{48}$

\section{Subgroup analysis}

No subgroup analysis is planned for our study. Studies reporting more than one outcome will be meta-analysed as per the same measure outcome. Any outcome shared by two or less studies will not be included in the final results and discussion.

\section{Sensitivity analysis}

Where appropriate, a rerun meta-analysis will be performed with the fixed effects models to explore the impact of smaller trials. Sensitivity analysis will also be scheduled subject to advice from a statistician.

\section{Management of research materials and progress monitoring}

All data and research materials in this study will be managed electronically, and data will be saved and password encrypted. To ensure accessibility across geographic domains, files will be uploaded to a trusted cloud-based platform. For data security, backup of electronic files will be made frequently onto duplicate sets of USB flash drives that will be kept by LL and one other reviewer in rotation. Correspondence for update and exchange of materials will be made electronically via encrypted email. Face-to-face meetings will be scheduled periodically once every 2 weeks for reporting and progress monitoring. Where physical presence is not possible, over-the-network meetings will be conducted.

\section{Ethics, knowledge dissemination and impact of study}

No ethics approval will be necessary for this meta-analysis. This study aims to provide a more updated and critical evaluation of the effects and efficacy of MBSR as a single intervention for chronic pain conditions that are not associated with somatising or cancer elements. On completion of the study, results will be disseminated to fellow researchers and medical professionals via peer-reviewed publications and international conferences. The reviewers anticipate that this study will provide better evidence-based guidance for healthcare stakeholders and policy setters in deciding whether MBSR is a cost-effective therapy for this category of chronic pain. 
Contributors LL initiated and conceptualised the research objectives and questions, completed the first round of electronic search, selection of publications and assessment of their eligibility and quality. The other three reviewers (HH, MM and JK) started collaboration with LL to revise the completed tasks and to pursue subsequent processes. LL drafted the manuscript for this protocol, receiving critical comments from the other reviewers.

Funding This research received no specific grant from any funding agency in the public, commercial or not-for-profit sectors.

Competing interests None declared.

Provenance and peer review Not commissioned; externally peer reviewed.

Open Access This is an Open Access article distributed in accordance with the Creative Commons Attribution Non Commercial (CC BY-NC 4.0) license, which permits others to distribute, remix, adapt, build upon this work noncommercially, and license their derivative works on different terms, provided the original work is properly cited and the use is non-commercial. See: http:// creativecommons.org/licenses/by-nc/4.0/

\section{REFERENCES}

1. Ospina $\mathrm{M}$, Harstall $\mathrm{CH}$. Prevalence of chronic pain: an overview. 2002. http://www.ihe.ca/documents/prevalence_chronic_pain_0.pdf (accessed Nov 2014)

2. Argoff CE, Viscusi ER. The use of opioid analgesics for chronic pain: minimizing the risk for harm. Am J Gastroenterol 2014; 2:3-8.

3. Moulin DE, Clark AJ, Speechley M, et al. Chronic pain in Canadaprevalence, treatment, impact and the role of opioid analgesia. Pain Res Manag 2002;7:179-84.

4. Boulanger A, Clark AJ, Squire $P$, et al. Chronic pain in Canada: have we improved our management of chronic noncancer pain? Pain Res Manag 2007;12:39-47.

5. Choudhury Y, Bremner SA, Ali A, et al. Prevalence and impact of chronic widespread pain in the Bangladeshi and White populations of Tower Hamlets, East London. Clin Rheumatol 2013;32:1375-82.

6. Johnson MI, Elzahaf RA, Tashani OA. The prevalence of chronic pain in developing countries. Pain Manag 2013;3:83-6.

7. Melzack R. Evolution of the neuromatrix theory of pain. The Prithvi Raj Lecture: presented at the third World Congress of World Institute of Pain, Barcelona 2004. Pain Pract 2005;5:85-94.

8. Leung $L$. From ladder to platform: a new concept for pain management. J Prim Health Care 2012;4:254-8.

9. Deshpande A, Furlan A, Mailis-Gagnon A, et al. Opioids for chronic low-back pain. Cochrane Database Syst Rev 2007;(3):CD004959.

10. Chaparro LE, Furlan AD, Deshpande A et al. Opioids compared to placebo or other treatments for chronic low-back pain. Cochrane Database Syst Rev 2013;8:CD004959.

11. Chaparro LE, Furlan AD, Deshpande A, et al. Opioids compared with placebo or other treatments for chronic low back pain: an update of the Cochrane Review. Spine 2014;39:556-63.

12. Ventafridda V, Saita L, Ripamonti C, et al. WHO guidelines for the use of analgesics in cancer pain. Int $J$ Tissue React 1985; 7:93-6.

13. Takai $\mathrm{Y}$, Yamamoto-Mitani N, Abe $\mathrm{Y}$, et al. Literature review of pain management for people with chronic pain. Jpn J Nurs Sci 2014. doi:10.1111/jins.12065. [Epub ahead of print].

14. Makris UE, Abrams RC, Gurland B, et al. Management of persistent pain in the older patient: a clinical review. JAMA 2014; 312:825-36.

15. Kabat-Zinn J. An outpatient program in behavioral medicine for chronic pain patients based on the practice of mindfulness meditation: theoretical considerations and preliminary results. Gen Hosp Psychiatry 1982;4:33-47.

16. Kabat-Zinn J, Lipworth L, Burney R. The clinical use of mindfulness meditation for the self-regulation of chronic pain. J Behav Med 1985;8:163-90.

17. University of Maryland Medical System. Center for Mindfulness. Courses and Schedules Secondary Center for Mindfulness. Courses and Schedules. 2014. http://www.umassmed.edu/cfm/ stress-reduction/mbsr-8-week/

18. Pickert K. The art of being mindful. Finding peace in a stressed-out, digitally dependent culture may just be a matter of thinking differently. Time 2014;183:40-6.
19. Matousek RH, Dobkin PL, Pruessner J. Cortisol as a marker for improvement in mindfulness-based stress reduction. Complement Ther Clin Pract 2010;16:13-19.

20. Carlson LE, Speca M, Patel KD, et al. Mindfulness-based stress reduction in relation to quality of life, mood, symptoms of stress and levels of cortisol, dehydroepiandrosterone sulfate (DHEAS) and melatonin in breast and prostate cancer outpatients.

Psychoneuroendocrinology 2004;29:448-74.

21. Carlson LE, Speca M, Faris $P$, et al. One year pre-post intervention follow-up of psychological, immune, endocrine and blood pressure outcomes of mindfulness-based stress reduction (MBSR) in breast and prostate cancer outpatients. Brain Behav Immun 2007;21:1038-49.

22. Gallegos AM, Hoerger M, Talbot NL, et al. Toward identifying the effects of the specific components of Mindfulness-Based Stress Reduction on biologic and emotional outcomes among older adults. $J$ Altern Complement Med 2013;19:787-92.

23. Lengacher CA, Kip KE, Barta M, et al. A pilot study evaluating the effect of mindfulness-based stress reduction on psychological status physical status, salivary cortisol, and interleukin-6 among advanced-stage cancer patients and their caregivers. J Holist Nurs 2012;30:170-85.

24. Witek-Janusek L, Albuquerque K, Chroniak KR, et al. Effect of mindfulness based stress reduction on immune function, quality of life and coping in women newly diagnosed with early stage breast cancer. Brain Behav Immun 2008;22:969-81.

25. Creswell JD, Irwin MR, Burklund LJ, et al. Mindfulness-Based Stress Reduction training reduces loneliness and pro-inflammatory gene expression in older adults: a small randomized controlled trial. Brain Behav Immun 2012;26:1095-101.

26. Kilpatrick LA, Suyenobu BY, Smith SR, et al. Impact of Mindfulness-Based Stress Reduction training on intrinsic brain connectivity. Neuroimage 2011:56:290-8.

27. Lutz J, Herwig U, Opialla S, et al. Mindfulness and emotion regulation-an fMRI study. Soc Cogn Affect Neurosci 2014:9:776-85.

28. Haase L, Thom NJ, Shukla A, et al. Mindfulness-based training attenuates insula response to an aversive interoceptive challenge. Social Cogn Affect Neurosci 2014. doi:10.1093/scan/nsu042. [Epub ahead of print].

29. Wells RE, Yeh GY, Kerr CE, et al. Meditation's impact on default mode network and hippocampus in mild cognitive impairment: a pilo study. Neurosci Lett 2013;556:15-19.

30. Dunn BR, Hartigan JA, Mikulas WL. Concentration and mindfulness meditations: unique forms of consciousness? Appl Psychophysiol Biofeedback 1999;24:147-65.

31. Davidson RJ, Kabat-Zinn J, Schumacher J, et al. Alterations in brain and immune function produced by mindfulness meditation. Psychosom Med 2003:65:564-70.

32. Travis F, Arenander A. EEG asymmetry and mindfulness meditation. Psychosom Med 2004;66:147-8; author reply 47-8.

33. Kerr CE, Jones SR, Wan Q, et al. Effects of mindfulness meditation training on anticipatory alpha modulation in primary somatosensory cortex. Brain Res Bull 2011;85:96-103.

34. Berkovich-Ohana A, Dor-Ziderman Y, Glicksohn J, et al. Alterations in the sense of time, space, and body in the mindfulness-trained brain: a neurophenomenologically-guided MEG study. Front Psychol 2013;4:912.

35. Teixeira ME. Meditation as an intervention for chronic pain: an integrative review. Holist Nurs Pract 2008;22:225-34.

36. Veehof MM, Oskam MJ, Schreurs KM, et al. Acceptance-based interventions for the treatment of chronic pain: a systematic review and meta-analysis. Pain 2011;152:533-42.

37. The Cochrane Collaboration. Cochrane Handbook for Systematic Reviews of Interventions. 2011.

38. Liberati A, Altman DG, Tetzlaff J, et al. The PRISMA statement for reporting systematic reviews and meta-analyses of studies that evaluate healthcare interventions: explanation and elaboration. $B M J$ 2009;339:b2700.

39. Queen's University Library. Ovid Health and Life Sciences Database Service. Secondary Ovid Health and Life Sciences Database Service. 2014. http://library.queensu.ca/research/databases/record/2922

40. CADTH. GREY MATTERS. Secondary GREY MATTERS. 2014. http://www.cadth.ca/en/resources/finding-evidence-is

41. The Cochrane Collaboration. Data Collection Form-Intervention review for RCTs only. Secondary Data Collection Form-Intervention review for RCTs only. 2014. http://www.cochrane.org/sites/ default/files/uploads/forums/u389/ERC\%20data\%20collection\% 20form\%20for\%20intervention\%20reviews\%20for\%20RCTs\% 20only.doc 
42. The Cochrane Collaboration. RevMan 5 Download and Installion. Secondary RevMan 5 Download and Installion. 2014. http://tech. cochrane.org/revman/download

43. The Cochrane Collaboration. Table 8.5.d: Criteria for judging risk of bias in the 'Risk of bias' assessment tool. Secondary Table 8.5.d: Criteria for judging risk of bias in the 'Risk of bias' assessment tool. 2011. http://handbook.cochrane.org/chapter_8/table_8_5_d_criteria for_judging_risk_of_bias_in_the_risk_of.htm

44. Hedges LV, Olkins I. Statistical methods for meta-analysis. 1st edn. Orlando, FL: Academic Press, 1985.
45. The Cochrane Collaboration. The standardized mean difference. Secondary the standardized mean difference. 2011. http://handbook. cochrane.org/chapter_9/9_2_3_2_the_standardized_mean_difference.htm

46. Higgins JP, Thompson SG, Deeks JJ, et al. Measuring inconsistency in meta-analyses. BMJ 2003;327:557-60.

47. Ioannidis JP. Interpretation of tests of heterogeneity and bias in meta-analysis. J Eval Clin Pract 2008;14:951-7.

48. Ioannidis JP, Trikalinos TA. The appropriateness of asymmetry tests for publication bias in meta-analyses: a large survey. CMAJ 2007:176:1091-6. 